\title{
The Narrative Study on Healing of Autism Disorder by the Tran Message of Body
}

\section{Mee-sook Cheong, Yeon-jung Tae}

Section: Healthcare

Sci. Journal Impact

Factor: 6.1 (2018)

ICV: 90.90 (2018)

Department of Naturopathy, Dongbang Culture Graduate University, Seongbuk-dong, Seongbuk-gu, Seoul, Republic of Korea.

\section{ABSTRACT}

Introduction: This is a case study on children with Autism Spectrum Disorder (ASD). It is also a narrative study that expresses each subject well and describes them vividly. The purpose of this study is to help the children with ASD to lessen their pain and to heal them through Tran Message of Body, a method which can only be performed and possessed by unique individuals.

Aim and Objective: When a person with Tran Message encounters someone with disease symptoms, that person will automatically touch the affected area, diagnose the unhealthy or affected part of the body and empathize with it. In this study, clients are taught to recognize the physical, psychological, mental, and environmental problems of their health and helped them to practice self-healing by recovering their inherent potential. According to the Diagnostic and Statistical Manual of Mental Disorders DSM5 , ASD is characterized by extreme unresponsiveness to others, severe communication deficits, overly rigid and repetitive behaviours, interests, and activities. Narrative research is a way to find the meaning of experiences in the process of talking about and retelling the experiences with which human beings meet in life and to study the process of living a new life based on this. It is also the most appropriate research method for capturing, interpreting, and healing the client's experiences in the context of time, place, and sociality as the client and counsellor interact in the psychological counselling area.

Conclusion: This study found that although the disorder is the same, the cause and the healing principle are different. Healing through Tran Message of the body are thought to be more sophisticated and a perfect healing method because it recognizes and solves the fundamental problems that each client has. However, this has limitations because the number of participants is limited to three. More systematic and continuous research is needed within this area of study.

Key Words: Tran Message of the body, Healing, Autism, Case study, Narrative

\section{INTRODUCTION}

In 1733, the British physician Chain (G. Cheyne) in his book, The English Malad, wrote that 'one in three' or more among people who lived in the golden age did not reach a fatal stage. He said that he suffered from illnesses of the body and mind because of severe discord or fancy. Illnesses have been argued to exist due to unhealthy lifestyle, poor urban planning, daily life lacking humour, food intake with high nutritional value, and population growth and its density ${ }^{1}$. Looking at the cause of mental illness, which Chain pointed out about 290 years ago, today's mental illness is not much of a difference.

In the development of modern medicine, human beings have been freed from the fear of disease. Nevertheless, the number of neurotic incidences is not declining to reduce the neurotic incidence, diseases which are difficult to cure with modern medicine have been considered for natural healing therapies ${ }^{2}$.

One of these methods is the Tran Message of body ${ }^{3}$, which is possessed by unique individuals. When a person with Tran Message encounters someone with disease symptoms, that person will automatically touch the affected area, diagnose the unhealthy or affected part of the body, and empathize with it. The symptoms revealed with the disease can be traced to any energy disorder. The person will be able to recognize details of the pain e.g. what causes it and through what method it can be healed. In this study, clients are taught to recognize the physical, psychological, mental, and environmental problems of their health. They are also taught to practice self-healing by recovering their inherent potential.

\section{Corresponding Author:}

Mee-sook Cheong, Department of Naturopathy, Dongbang Culture Graduate University, Seongbuk-dong, Seongbuk-gu, Seoul, Republic of Korea; E-mail: ayurveda16@naver.com

ISSN: 2231-2196 (Print)

Received: 15.06 .2020
ISSN: 0975-5241 (Online)

Revised: 10.07 .2020
Accepted: 18.07 .2020 
This is a narrative study on children with ASD and how the Tran Message helped them heal. The term ASD has been used since the 1990s. According to DSM- $5^{4,5}$, it is a developmental disorder ${ }^{6,7}$ such as social interaction disorder, communication disorder, speech disorder, behavioural disorder and a state lost in his world because of intellectual and cognitive deficits. ASD is characterized by extreme unresponsiveness to others, severe communication deficits, overly rigid and repetitive behaviours, interests, and activities.

The cause of ASD is the failure to develop mind theory. Mind theory is the perception that people act based on their beliefs, intentions and mental states, and not on information. ${ }^{8,9}$ More recent studies in the biological and psychological domain have led clinical theorists to think that cognitive problems and brain abnormalities are the primary causes of autism ${ }^{10}$.

The fact that childhood experiences have a profound effect on an adult's mental health and that children are more compliant than adults is easy to treat, and it is positively accepted by psychologists, behaviourist psychologists, and biologists ${ }^{11}$. Therefore, the causes, treatment, and prevention of childhood mental disorders have great significance in the study of autism spectrum disorders.

Relatively recently, as technology and theory have become the basis of scientific research models, psychologists have introduced experimental methods to test their hypotheses. Even clinicians and researchers who prefer technical methods tend to take the method of scientifically analyzing the case data obtained from patients by collecting it objectively rather than interpreting it according to their tastes with knowledge gained from clinical intuition.

As described above, ASD is a neurodevelopmental disorder according to DSM-5, which integrates past childhood autism, childhood autism, high function autism, childhood decay disorder, Asperger's disorder, and other general disorders into ASD.

In this trend, Narrative ${ }^{12}$ research is a way to find the meaning of experiences in the process of talking about and retelling the experiences with which human beings meet in life and to study the process of living a new life based on this. It is also the most appropriate research method ${ }^{13}$ for capturing, interpreting, and healing the client's experiences in the context of time, place, and sociality as the client and counsellor interact in the psychological counselling area.

Narrative research tells stories that are more than just an enumerated and told the story. It is a story with a meaningful life. It retells and revises the story while telling the story, and creates a new story ${ }^{14}$. This study creates a narrative that tells the story of an Autism child with a disability who had difficult life experiences and was healed through the Tran Message of the body.
Looking at previous studies, as a narrative role, 'alternative narrative' or 'experiential narrative' is selectively borrowed and written. Da-Hyun $\mathrm{Kim}^{15}$, Young-soon Jeong ${ }^{16}$, and Deok-in $\mathrm{Cho}^{17}$ follow the 'experiential narrative' application method.

Kim Gwang-ho ${ }^{18}$, Woo-hyeok ${ }^{19}$ is composed of descriptive, explanatory and healing narratives, and allows research participants to discover new personal and social perspectives, talk and organize narratives, showing the changes and effects of life as an alternative in the healing narratives. In this study, the term "healing narrative" is considered to be more meaningful as a more specific and behavioural action than the term "experiential narrative". It was descriptive as a healing narrative and reflected in the research text.

In this study, it can be observed that the cause of occurrence is different for each person even with the same disorder ${ }^{20}$. Just as the constitution, environment ${ }^{21}$, and personality are different, the effect of the healing reaction is also different according to each person's uniqueness and consciousness ${ }^{22}$, ${ }^{23}$.

Tran Message of the body is where the human being is connected to the body, mind, and soul. Rephrase. The healer hears a sound (the Tran Message) of the body, and then heals the person with balance. A study of this method is rare. Therefore, it is necessary to study the Tran Message of body. This research is the purpose to understand further that this method eases the pain of children with ASD and thus heal them.

\section{STUDY METHOD}

\section{Study plan}

This study was conducted from March 2019 to December 2019. The healing period was from May 1, 2019, to October 31,2019 , a total of six months. This study was conducted on three subjects with autism disease at the Tran Message of body Institute located in CH City. It was qualitatively analyzed as a narrative study suitable for the characteristics of this study.

\section{Study participants}

The initial numbers of participants recruited for the study were seven. Two of them were excluded because the individual's disease did not match the study. Another two participants went to the study together but were excluded due to unfaithfulness.

Participants in this study were three males aged 6, 15, and 18 with a common disability of ASD. They were continuously treated by modern medicine but later shifted to a research centre offering Tran Message because they find the earlier 
method ineffective. Since the study participants were all minors, the study was conducted after explaining the contents of the study to their parents as well as after getting their participants' approval.

\section{DATA ANALYSIS}

This is narrative research based on subjective interpretive epistemology. It studies and interprets the unmanipulated words or behavioural trace of the study participants and finds the meanings of their behaviour. Narrative research provides a framework for thinking about experience in a three-dimensional space that is not only a phenomenon but also a situation, continuity, and interaction.

As a form of inquiry that seeks to understand human experiences, it focuses on understanding humans and understanding human experiences before understanding the diseases that give meaning to the basic structure of experience. The experience of pre-language trauma is unrecognizable and unrememberable, which gives unity to the unclearness of experience in the three-dimensional space of the researchers and the participants.

This study was conducted according to the narrative exploration procedure suggested by Clandinin and Connelly. The whole research training course of narrative exploration method is made up of at first 'entering into the field ', second, 'from field to field text ', third, 'composing field text ', fourth, 'from field text to research text' and fifth, 'Write the text of research'. However, since these processes are not strictly classified, "field text in the field" and "composing the field text" can be integrated as steps for collecting data. Also, 'study text in field text' and 'writing research text' can also be integrated into a step of data analysis. Therefore, in this study, the narrative research procedure was composed of three steps: 'entering the field', 'composing the field text', and 'composing the research text'.

\section{STUDY RESULT}

\section{Descriptive narratives (Symptoms)}

The three participants had severe autism spectrum disorders as indicated by their hospital certificates. They were characterized by extreme unresponsiveness to others, severe communication deficits, overly rigid and repetitive behaviours, their interests, and activities.

The 6-year-old participant was a kindergartner brought by his mother. The outwardly neat and handsome participant neither made eye contact nor spoke as soon as he sat down. Even though his mom gave him some candy, he could not unwrap it. His mom told the counsellors that one month after her child entered kindergarten, she was told to take him back home because he was autistic.

The 6-year-old participant has been determined to have an untreatable disorder not only from Korean traditional medicine but also from occidental medicine. His mom said that she worked as a teacher together with her husband and lived hard, but she did not know that her child had such a disorder and that she could not do anything for him. She also said that because she had to work together, her child was, since birth, reared by his grandmother, the oldest and the younger aunts.

The 15-year-old participant also visited the research center with her parents and appeared to be tall, thin, underweight, and weak. While sitting, he cast a side glance at the research center representative. Whatever they ask including his name, he just said a word 'yes'. Though he was asked his age, he answered 'Yes'. Though asked what his name was, he said 'Yes'. His parents said that they had a disability registration because they could not afford their with child's treatment.

His elder brother has suffered the physically handicapped autistic disorder. He also suffered from autism disorder but was more severe than the mentioned client. During longterm treatment, he has become the center of his family. The parents said their children often showed tears. They could not find any way to help their children that is why they came to the research institute

The 17-year-old participant looked enervated and had dull eyes. He was a long-term patient who had been treated for autism spectrum disorder for 17 years. But he was not in remission. Therefore, he visited the research center. He was diagnosed with the ASD, attention deficit, deficiency of language, cognition and social function, and had anxiety disorder as well.

The Participant had a small body size for his age and did not look healthy. He looked like a 14-year-old child. He was unable to study at school due to autism spectrum disorder. He was often subjected to negative, haunting comparisons with relatives and peers. Hence, he did not socialize with his friends and was living in isolation. $\mathrm{He}$ was always trapped in himself in this room. He did not with his parents, and he was not even talk.

As described above, ASD is a neuro developmental disorder according to DSM-5, which integrates past participant hood autism, participant hood autism, and high function autism and participant hood decay disorder. This should be Asperger's disorder and other general disorders into ASD.

\section{EXPLANATORY NARRATIVES (CAUSE)}

According to the diagnosis of the three participants of this study, they noticeably suffered from a lack of affec- 
tion from their parents. It seems that the experiences of their participant hood had a profound effect on the mental health of being adults.

From his birth onwards, the six-year-old participant had no opportunity to form an attachment to his parents because they were both dual-working. When the counsellor put her hand on the participant's chest, she found that his chest was cold and empty and had no connected energy. There was no mother in the mind of the child. He seemed to have no connection with any energy. To do something. All the energy lines did not work. The participant was trapped in the dark, had no idea, and had no power.

The parents changed their parenthood, therefore changing the parenting environment. The participants found themselves closing their minds and words by emotional trauma. They did not meet their basic needs during the development process and stopped expressing their intention to complain. It was found that the cause of the six-year-old participant's autism was due to environmental factors and no communication with their parents.

The Tran Message of the body of the Participant said that they wanted to be loved by their parents. In particular, the counsellor told his mother to quit his job and to spend more time with her child. The counsellor encouraged her to act as a mother so that he would feel true motherhood, not only relying on money as a form of parenting. The counsellor also said that the mother should be healed as well.

The 15-year-old participant seemed to have the tangle of family relations in the Tran Message of the body as soon as he made eye contact with counsellors. The tangle was connected to the original family, but the 15-year-old participant was nowhere in the entangled family. The counsellors looked for him and found him sitting in a corner. The participant became the body of an ancestor who was not loved. He became lethargic, possessed by a spirit of a sad ancestor.

Maybe, because of the long illness of his elder brother, his parents' interests were biased toward him. Therefore, it seemed that his self-obliteration and anger drove him to enervation. The counsellor's hand went to the participant's neck and upper back, which were stiff. The participant was rigid and there was no receptive energy throughout the body.

When the participant was only about six to seven years old, his parents only gave intensive care to his older brother. While the participant was eating, the mother said, 'brother has to eat this.' So, the participant could not even eat as he wanted. As time passed, curiosity about food disappeared and words disappeared. The participant had itself deep in his heart anger toward his parents due to severe discrimination from his older brother. The participant went into a dark cave without realizing his anger. He gave his body to their ancestor, eventually becoming autistic disabled
The counsellor was able to confirm that the cause of Autism disorder was psychological.

The counsellor put her hand on the hand of the 17-year-old participant. The Tran Message from his hand let her feel the shock and fear which he experienced in infants. She also felt that all the energy of his body was blocked. The blockage of energy seemed to prevent all the functions from developing. The head, body, arms, and legs are blocked. The disorder shown as autism was not a cause but a result. And if what the body wants was solved, the disorder was not already a disorder.

The participant was tied to the trauma he received during infancy. The counselor received information stored in the participant's body (trauma received during infancy) through resonance. The participant then stopped the development of all functions due to shock and fear. He was obsessed with tearing clothes off as a defence mechanism. His parents punished him without any good talks afterwards. The counsellor felt a startled Kkamjjak Kkamjjak surprise in a loud voice from the participant's hand. Because the parents did not know this, their child became resentful and upset. When the participant was an infant, the couple fought loudly and said they did not remember the cause.

The participant strongly resisted going to treatment after being diagnosed with autism disorder in the hospital. When the counsellor's hand gripped the participant's hand, the Tran Messages of the body was heard from his hand. The participant strongly resisted said he wanted to be free from fear. He said he wanted to be a normal person. The counsellor asked the parents to tightly hug the participant from both sides. When the parents hugged the participant, he cried without hesitation. When the mother touched the participant's chest and said she was sorry, the participant's body began to warm. This participant also found that the causes of his autism disorder are psychological and emotional factors.

As such, it was found that the disability that appeared to be autism disorder was not a cause but a result. The reason for the disability is that the body does not know what it wants. If we notice the Tran Message of the body, we may have less disability.

\section{HEALING NARRATIVES}

In conclusion, all three participants needed to restore trust with the primary caregiver or the mother. The participant's ren had to build their faith in the world and their identity.

When the counsellor's hand touched the chest of the 6-yearold participant, a "cor-reok cor-reok" began to sound from his chest. The participants began to breathe as soon as her chest sounded. The participant made a profound sigh. As the counsellor touched the participant's chest and back, the body 
warmed up, and the participant began to relax. And fell into a deep sleep. The counsellor told the parents while the participant was asleep.

The counsellor made his mother take time off from work and care for her participant so that he could accept her and recover the family relationship. He received treatment with his mother and consciousness of his mother was also corrected through healing for two months. In his consciousness of mother, the frame of subjective judgment and irrational belief changed rationally. As the LUX of the consciousness of mother rose, the participant also healed. As the participant had a good circulation of energy, he was able to breathe normally. Through interactions with the main caregiver, the development has been normalized in five parts (language, body, cognition, emotion, and society).

The parents learned that a stable attachment to the main up bringer immediately leads to trust, and as trust is restored, it leads to faith in the world. And the counsellor's hand was purified according to the message given by the participant's body, mind, and spirit. Also, the participant recovered life energy through a stable recovery of trust. The participant's closed-chest was opened and recognized the existence of a mother, and it was possible to develop with faith toward the world in addition to expressing his basic needs. Feeling the love of the mother, the life energy of the participant was revived, and emotional trauma was resolved. As healing begins, Participant started speaking. And the mother of the participants liked it more. The participant's mother began to excite the words without hesitation.

\section{Case 1:}

"Teacher, my participant started talking."

"It is strange, so I feel like I'm going crazy."

"Last week, I played by touching it by my side."

"Teacher, My child expresses emotions."

"Eomeo, Eomeo, Thank you!"

"Oh, my gosh! I didn't know it would be different. Thank you, thank you."

The participant was poor at expressing language. As the participant started expressing, his expressions also changed. The participant started acting and expressing emotions.

\section{Case 2:}

"Mom, don't go anywhere!"

"Mom, do you love me?"

"Daddy, eat something delicious with mom!"

"We are together, right?"

"Hehehe"

"I'm shy."

"Mom--, Mom---"
"Go- - -"

As with the 15-year old participant, when the counselor touched his neck, his neck softened and noticed a warm energy flowing.

The 15-year-old participant found himself in a tangle of family relationships. He was given new awareness to family relationships so that all of them could play a right role in family relationships. Along with breathing, energy flowed throughout their body from head to toe. As energy flowed, each body could function smoothly. The energy of languagerelated functions (body organs such as the brain, neck, mouth, heart, lungs and kidneys) can play their own role too. The body organs were able to produce a clear sound wave and express their own minds and feelings in language.

When counselor kicked out, the ancestral spirits in the participant's body, the participant recovered by, purifying the body through breathing. The participant, who had not eaten anything, gradually found his appetite. Due to normal food intake, he overcame eating disorders and regained his confidence by developing inner power. Besides, as he restored a family relationship with his parents and elder brother, the love between the family members also grew.

As the participant recovered, the mother's voice changed, and a bright smile spread across her face. She is also a great talker.

\section{Case 3:}

"Teacher! My child has changed."

"I did not even see my eyes, but they looked at me."

"When I see it, the child seems to be lighter."

"It's the first time my child is laughing."

"It is so good in the world"

"Teacher, how can we not even have a big child?"

"I'm sorry for asking too much. You said no last time. .."

There was no action but he started to express himself. It was seen that the participant wanted to express something so bad.

\section{Case 4:}

"I can't see my grandfather who was holding hands with me."

"Because my grandfather is invisible, my eyes are bright."

"Mom! Mom! You are my mom, right?"

"What I want to eat is rice!"

"You're warm at heart!"

"Brother, go together with us!" 
"I want to play with my friend, too."

"I should eat with my brother. It's good for me."

The counsellor's hand touched the head of the 17-year-old participant, and the tight energy began to flow. Then, the counsellor's hands moved back to his chest but there was still no great flow of energy. As the hand of the counsellor went to the arms and legs to give warmth, energy began to flow slowly. In the case of the 17 -year-old participant, his perception of parents must be changed. They realized that their son was treated as an autistic participant for 17 years and why he abnormally perceived and resisted them.

It helped them changed. As the participant's energy flows well, the brightness of his consciousness (LUX) also increased. The energy of shock experienced at that time flowed out with a large amount of urine. They cancelled the disability registration of their son as his body told the counsellors. While the counsellor put her hand on the participant's chest and back, the fear tied with the subconscious was healed. His defence mechanism was no longer used.

The healing began when the parents realized that they were responsible for their participant's autism disorder. The counsellor stroked the participant's back and said it was okay, "You are OK". The participant seemed to receive energy as the frozen body slowly melted. The energy line of the participant opened and felt a strong shudder, and soon became quiet.

The parents felt guilty that their participant had problems because of their disharmony. As the participant began healing, the parents were relieved and grateful.

\section{Case 5:}

"Thank you. Thank you."

"It's like a dream to be treated like this."

"I knew for the first time that everything was our fault."

"There were too many things we did wrong."

"Forgive the baby."

As a smile appeared on the participant's face and have vitality, he tried speaking. Since he lived without expression, it seemed difficult to express. However, through healing, he does not seem to have difficulties at all.

\section{Case 6:}

$$
\begin{aligned}
& \text { "I'm shy!" } \\
& \text { "I'm free from disability!" } \\
& \text { "I'm light enough to fly in the sky!" } \\
& \text { "Thank God, I didn't die!" } \\
& \text { "I'm hungry!" } \\
& \text { "Mom, I love you!" } \\
& \text { "Thank you!" }
\end{aligned}
$$

In healing, the disorder is the same, but the cause is different, therefore, the healing principle is also different. The healing principle is appropriate to the trauma of each client.

\section{DISCUSSION}

This study is a case study of healing autism through the Tran Message of the body. There is no research yet on healing with the Tran Message of the body that sees the human body, the mind, and the soul as it is listened to the Tran Message of the body, and heals according to the sound. Therefore, it is necessary to study this method. The purpose of this study is to help the participant's ren with autism spectrum disorders heal.

As a result of this study, it was found that external factors were affecting ASD such as environment, and internal factors including psychology, and the other factors such as biological and potential unconsciousness, etc. It was also found that all the participants were not aware of the factors and causes and concentrated on treating only the symptoms revealed by the disorder. Therefore, this study is aimed at their 'bodies' signals and show them what they do not recognize and to respond accordingly to heal.

Through the sound of the body, the anxiety about the relationship with their parents was confirmed that of the jape of participants aged 6,15 , and 17 . It was proved that there is no hope in life without faith. We can also find that empathy gave humans the power to tear down even the thickest and hardest parts. The energy of healing for the participants is Empathy and understanding.

This result can also be seen in the study of Jang Woo-hyuk, which suggests that the healing narrative coincides with the stage of healing through the principle of meditation. The process of deriving the results as a narrative study can be considered to be consistent with the studies of Kim Da-Hyun, Jeong Young-soon, Jo Deok-in, and Kim Kwang-ho.

The healing by the Tran Message of the body not only heals the physical body but also the mental. Someone diagnosed with the same disease can have other symptoms and the healing effect can also be different. Before knowing about the world, and even before learning to talk, the participant with autism spectrum disorder was connected with his parents' thoughts, minds, and consciousness. It was later affected by his parents. Therefore, such an influence led to the loss of balance and appearance of the symptom. So, prelinguistic thought and mental expression are autistic symptoms.

In general, there is no way to guarantee a complete cure for autism spectrum disorder. There are just individualized special education, speech therapy, behavioural modification therapy, and medication, which are long-term supplementary treatments. 


\section{CONCLUSION}

We could confirm that the cause of autism disorder was psychological. While this method recognizes and solves the fundamental problems of each client, we think that the healing by the Tran Message of the body is more sophisticated and tailored healing. However, we continuously need to study systematically in this field.

Acknowledgement: Authors acknowledge the immense help received from the scholars whose articles are cited and included in references of this manuscript. The authors are also grateful to authors / editors / publishers of all those articles, journals and books from where the literature for this article has been reviewed and discussed.

Funding: None

Conflict of interest: None

\section{REFERENCES}

1. Lee HS. Psychology of abnormal behaviour. Seoul: Dae Wang Sa. 1985.

2. CheongM.S, Y. J. Tae. Case study on the healing of autism disorder by the tran message of body. Journal of community healthcare and development. 2020;1(1):7-12.

3. Y. J. Tae. The Tran Message of body heals. Tarea. 2015:23.

4. U. K. Lee. Abnormal Psychology: DSM-5 update. Seoul. hakjisa. 2017:60-65.

5. J. C. Ronald. Abnormal Psychology. New York. Worth Publishers. 2014:515-516.

6. H. S. Lee. Psychology of abnormal behaviour. Seoul. Daewangsa. 1999:7.

7. M. H. Shin, Y. H. Seo, S. J. Song, Y. G. Kim, Y. S. Won, W. G. No, J. M. Kim, S. Y. Kang, H. Y. Lim. Developmental Psychology. Seoul: hakjisa. 2013:272.

8. Frith U. Cognitive explanations of autism. Acta Paediatrica. 1996 Oct;85:63-8.

9. Pisula E. The autistic mind in the light of neuropsychological studies. Acta neurobiological experiments. 2010.
10. Clandinin DJ, Connelly FM. Narrative inquiry: Experience and story in qualitative research.

11. Jeong HO, Kim YS. North Korean women defectors in South Korea and their political participation. International Journal of Intercultural Relations. 2016 Nov 1;55:20-31.

12. U. H. Jang. Healing Experiences of Middle-Aged Women from Dysfunctional Families: A Meditation Counselling based Narrative Study. Dongbang University PhD Thesis. 2018.

13. Y. S. Jeong. The Hwa-byung Healing Experience of Elderly Women: A Narrative Study. Dongbang University PhD Thesis. 2015.

14. D. I. Jo. Healing Experiences of Married Women Undergoing Generalized Anxiety using Reflected Image Meditation(RIM): A Narrative Study. Dongbang University PhD Thesis. 2016.

15. K. H. Kim. Female North Korean Women Defectors' Experiences of Healing from Family Dissolution: A Narrative Study. Dongbang University PhD Thesis. 2016.

16. M. S. Cheong and H. J. Jeon. A Study on the Improvement of Self-directed Learning Ability of Adolescents Using Mantra Meditation Program. Asia-Pacific Journal of Educational Management Research. 2018;3(1):27-32.

17. M. S. Cheong and M. J. Go. The Development and Effect of Âyurvedic Astrology Path Counseling Program. International Journal of Beauty Science and Technology. 2017;1(2):13-18.

18. M. S. Cheong and B. H. Jin. The Effect of Ayurvedic Color Therapy Program on Infant Attention Deficit. International Journal of Participant Warfare Promotion and Management. 2018;2(1):7-12.

19. M. S. Cheong and A. L. Rim. A Study on the Development of the Ayurvedic Types Scale. International Journal of Art and Culture Technology. 2018;2(1):7-12.

20. J. S. Yeom. Narrative Inquiry in Educational Research: The Concept, Procedure, and Dilemmas. Korean Journal of Anthropology of Education. 2002;6(1):119-140.

21. S. J. Choi, and M. J. Hwang. A Study on Moral Injury of Gwangju Democracy Movement. Asia-pacific Journal of Multimedia Services Convergent with Art, Humanities, and Sociology. 2019;9(8):735-748.

22. M. S. Cheong and Y. J. Tae. Case Study of Healing by the Tran Message of Body for ADHD. Asia-pacific Journal of Convergent Research Interchange. 2020;6(4):47-55.

23. D. H. Kim. The Meditation Counseling Experience of Adolescents with School Maladjustment: A Narrative Study. Dongbang University PhD Thesis. 2016. 